\title{
SERUM ANTIBODY TITRES FOR LEPTOSPIRA AND ITS ASSOCIATION TO THE IMMUNIZATION AND REARING PATTERN IN A SAMPLE OF DOGS FROM SUBURBS OF COLOMBO: A PRELIMINARY STUDY
}

\author{
M.G.Thammitiyagodage ${ }^{1}$, P. Somaratne ${ }^{1}$, M.A. Roshan Priyantha ${ }^{2}$, K.C.R. Perera ${ }^{1}$ \\ ${ }^{1}$ Medical Research Institute, No. 527, Dr Danister de Silva Mawatha, Colombo 8 \\ ${ }^{2}$ Veterinary Research Institute, P.O. Box 28, Gannoruwa, Peradeniya
}

\begin{abstract}
SUMMARY: Leptospirosis is an important zoonotic disease in man and animals. Increasing numbers of human Leptospirosis are reported each year in Sri Lanka. Rodents are most often the natural maintenance host of leptospira, but the potential of transmitting the disease from ruminants and dogs to human should not be overlooked. Aim of this study was to determine the serum antibody titres for leptospira in 139 dogs in selected suburbs of Colombo, Sri Lanka and evaluate its association with dog rearing pattern and immunization history.A questionnaire based survey was conducted to collect data on dog rearing pattern and vaccination history of dogs. In addition, the potential transmission of leptospira from dog to human and public awareness on canine leptospirosis was also assessed. Blood samples obtained from dogs were subjected to Microscopic Agglutination Test (MAT) to detect leptospira antibody levels and to identify respective strains. Strains were identified against 12 serovars of pathogenic genomospecies Leptospira interrogans. According to MAT results, around $62 \%$ of unvaccinated domestic dogs enrolled in the study had leptospira antibody titres ranging from 1:100 - 1:3200, indicating an acute or convalescent stage of infection. Moreover, 25\% of primarily vaccinated dogs, $17.9 \%$ of regularly vaccinated dogs and $36.8 \%$ of randomly vaccinated dogs failed to seroconvert. Serum samples subjected to sero grouping were all found to be within pathogenic genomic species Leptospira interrogans. Single sero groups $L$. canicola, $L$. australis, $L$. Icterohaemorragica and $L$. djsiman and mixed sero-groups $L$. australis and $L$. canicola were predominantly observed. No clinically proven Leptospirosis was reported among the owners of the dogs, their family members or other household pets even if they had close contact with their dogs. Among the surveyed population, only one person was aware of $(0.7 \%)$ possible transmission of leptospirosis from dogs. Statistically significant number of primarily vaccinated dogs was in indoor habitats and randomly vaccinated dogs were in outdoor habitats $(p<0.05)$. That trend was not observed in regularly vaccinated and unvaccinated dog population $(p>0.05)$. Leptospirosis being a highly infectious zoonotic disease, outdoor living unvaccinated and randomly vaccinated domestic canine populations may contribute to increasing incidence of Leptospirosis in man as well as in other domestic mammals.
\end{abstract}

\section{INTRODUCTION}

Leptospirosis refers to a range of disease conditions in man and animals caused by the spirochetes belonging to genus Leptospira (Evangelista and Coburn, 2010). It is an important zoonotic disease occurring worldwide (Radostits, 1989). There are saprophytic and pathogenic leptospira species. Pathogenic leptospira can be classified into Leptospira interrogans with more than 250 serovars and Leptospira borgpetersenii (Adler et al., 2011; Bulach et al., 2006). Strains or serovars are identified based on the characteristics of their surface proteins.

Leptospirosis is considered as an emerging public health problem worldwide due to increasing incident rate in humans living in urban settings(Evangelista and Coburn, 2010; Vijayachari et al., 2008). It is primarily considered as an occupational hazard to man. Increasing number of leptospirosis cases are reported every year in Sri Lanka among human beings (Victoriano et al., 2009) but, the real disease burden in animal population is not evaluated in the country.

The leptospires are normally carried in the kidney tissues of rodents and other small mammals and are excreted in their urine (Greenwood et al., 1992). The species in which infection is endemic and transferring from one animal to another animal by direct contact are considered as maintenance host. The disease is maintained as a chronic infection in the renal tubules of the maintenance host. Humans as well as animals can be accidental (incidental) host of leptospirosis. Animals may be maintenance hosts of some serovars but incidental hosts of others. If a maintenance host for a particular leptospiral serovar is infected with another serovar it may develop symptoms (WHO, 2003). Dogs are the maintenance host for the serovar, L.canicola (WHO, 2003). The maintenance host ensures the continuous circulation of a particular leptospiral serovar in a geographical area even without incidental hosts (WHO, 2003).

Corresponded auther: M.G.Thammitiyagodage, drmayuri.geetha@gmail.com 
Leptospirosis is very common in areas or seasons where climate is warm and humid with abundance of alkaline soil and water (Radostits, 1994). When there are favourable climatic conditions for the organism, animals as well as human beings can get the infection. As in humans, animals that are incidental hosts may become ill with severe disease. Mild leptospiral infection in domestic animals may remain unnoticed.

In all domestic animals, clinical manifestations of leptospirosis vary from acute to sub-acute and chronic infection. Chronic infection is localized in the kidneys (sometimes also in the genital tract) usually without detectable clinical evidence of illness. Animal carriers may shed leptospires for months or years in their urine, sometimes in very large numbers, contaminating moist soil or sources of drinking water (Radostits, 1994). The pollution of surface waters poses a risk of transmission of infection to other animals and humans, resulting in water borne outbreaks of infection in humans.

Leptospira interrogans has multiple serovars that could infect dogs. Infected dogs may suffer from a chronic disease leading to kidney damage, or may develop an acute Weil's-like disease syndrome after infection with certain serovars (Nelson and Couto, 2004). Previously infected animals develop antibodies and usually eliminate the organism quickly to remain sub clinically infected. Organism has the ability to multiply in different tissues of non immune host or animals that are infected with nonhost adopted species(Nelson and Couto, 2004). Clinical manifestations of leptospirosis in dogs are variable, as in other species of animals, can range from a total lack of symptoms (renal carrier state), which is most common, to severe icterohaemorrhagic disease with acute interstitial nephritis (Sykes et al., 2011). Unvaccinated puppies (aged up to 1 year) suffer from the most severe forms of the disease, sometimes with fatal outcome.

Annual vaccination in dogs can prevent the infection caused by specific serovars indicated in the vaccine and can be recommended for dogs at risk. In Sri Lanka dogs are generally vaccinated against $L$. Canicola and $L$. icterohaemorragica specific bi-valant vaccine. This vaccine is given as a primary vaccine when the dog is less than three months old and subsequently as a regular annual booster.

Dogs infected with leptospires may directly transmit the disease to humans or do indirect transmission through environmental contamination (Barragan et al., 2017). There are no published data available on sero prevalence of leptospirosis in domestic and stray dog populations in Sri Lanka. This is a preliminary study to assess the antibody titres for leptospira in vaccinated and unvaccinated dogs in suburban settings in Colombo. A questionnaire based study was conducted to identify the dog keeping pattern and immunization history. Final objective is to determine the predominant strains of leptospires in the selected canine population.

\section{MATERIALS AND METHODS}

Questionnaire based survey was conducted at six randomly selected private veterinary clinics located in the district of Colombo from January to August in the year 2012and part of the results were presented as an abstract form in the year 2013. Further, serological investigations and data analysis were done subsequently. Convenient sampling method was used for data collection. A total of 139 dogs aged between three months to 15 years were recruited for the study. The veterinary surgeons in private veterinary clinics filled the questionnaires by interviewing the owners of the dogs enrolled in the study. Information on the age of the $\mathrm{dog}$, sex, and vaccination history, any past medical history of laboratory confirmed leptospirosis in selected dogs were also collected. In addition, pet keeping habits of the owners with particular emphasis on closeness of the association with the dogs (keeping dogs inside or outside the dwellings) and the awareness on transmission of leptospirosis from dogs and any incidence of leptospirosis in dog owners or their families were evaluated. Parallel data collection was done in all the selected private veterinary clinics. Statistical significance of dog rearing and vaccination patterns were calculated by Chi square test using the SPSS statistical package.

\section{Collection of Blood samples from dogs}

Venous blood samples $(2 \mathrm{ml})$ were drawn from each animal aseptically, after obtaining informed consent from the owner. Blood samples were allowed to clot and centrifuged at $3500 \mathrm{rpm}$ for 15 minutes at room temperature to separate serum.

\section{Microscopic Agglutination Test}

Microscopic Agglutination Test (MAT) detects both IgM and IgG antibodies and was conducted using the standard method practiced at the Medical Research Institute, Colombo. Serum samples were incubated at $56^{\circ}$ $\mathrm{C}$ for 30 minutes to inactivate any non-specific antibodies present. A series of diluted serum was mixed with a suspension of live non-pathogenic Leptospira biflexa Patoc strain in microtitre plates and incubated at $30^{\circ} \mathrm{C}$ for 2 hours. The results were read under dark-field microscopy to identify the last dilution with $\geq 50 \%$ agglutinated leptospires. Titres reported $\geq 1$ : 100 were considered as positive. After performing MAT, only 43 serum samples were adequate enough to carry out the strain specific MAT test. Strain specific MAT was performed against 12 serovars of pathogenic strains of Leptospira interrogans including L. pyrogens, L. gem, $L$. ceylonica, L. australis, L. tarrasovi, L. pomona, L. hardjo, L. canicola, L. ratnapura, L. weerasighe, $L$. icterohaemorragiea and L. Djisimea at the Veterinary Research Institute, Gannoruwa, Peradeniya following the standard protocol given for (both qualitative and 
quantitative) selected serovar of leptospira described in manual of WHO/FAO reference laboratory on leptospirosis (Smythe, 1993).

Ethical clearance for this study was obtained from the Ethics Review Committee of the Medical Research Institute, Colombo on $2^{\text {nd }}$ August 2011 and ethics review number is $15-2011$.

\section{RESULTS}

Out of the surveyed population (139) $58.3 \%$ had received at least a single dose of a combined vaccine (DHL) containing leptospira, while remaining (41.7\%) dogs have never been vaccinated against the disease Vaccinated dogs were categorized in to four main groups for the purpose of data analysis, i.e. (i) dogs less than one year and given primary vaccination and subsequent booster, (ii) regularly vaccinated dogs (iii) randomly vaccinated and (iv) unvaccinated. Results of the percentage of dog population in different subgroups with their antibody $(\mathrm{Ab})$ titres are summarized. Out of the dogs that received primary immunization, $(25 \%)$ had no positive $\mathrm{Ab}$ titres by the time of testing. Majority of the primarily vaccinated dogs $(60 \%)$ had antibody titre in the range of 1:100 - 1:200 whilst very small fraction (15\%) had antibody titres as high as 1:1600. Among the dogs that have been regularly immunized against leptospirosis, (17.94 \%) did not have any immune response. Of them $72.1 \%$ of the dogs had antibody titres in the range of 1:100 - 1:200,7\% had antibody titres in the range of $1: 400-1: 800$ and $2.3 \%$ of the population has antibody titres as high as 1:1600 $1: 3200$. Few dogs that were over 10 years and regularly vaccinated showed very high antibody titres $(1: 800 \&$ $1: 3200)$. In the surveyed population, (15.7\%) were randomly vaccinated and of them $36.8 \%$ have not had positive titres against leptospira when tested. Only one $\operatorname{dog}(5.2 \%)$ had antibody titres as high as 1:400 whilst all the other dogs $(58 \%)$ had titres in the range of 1:100 $1: 200$. Out of the survey population $(41.7 \%)$ were never been vaccinated against leptospira but $(62 \%)$ of those dogs had antibodies against leptospira. Titres were in the range of 1:100 - 1:3200. Some dogs had antibody titres $>1: 400$ indicating either acute infection or recovering from infection.

None of the dog owners or their family members had clinically proven leptospirosis and there was no proven evidence of leptospirosis among their pet dogs. Of the surveyed population only one person knew that the domestic dogs are possible source of human leptospirosis.

According to the questionnaire based study, $48.9 \%$ of dogs were kept inside the houses, whilst $51.1 \%$ were in the garden or outside (Table 1).

Serum samples were subjected to sero grouping and were all found to be within pathogenic genomospecies Leptospira interrogans. Some samples were of single serogroup namely L.canicola $(\mathrm{n}=12)$, L. australis $(\mathrm{n}=2)$, L. Icterohaemorragica $(\mathrm{n}=1)$, and L. djsimea $(\mathrm{n}=1)$ whilst some serum samples were positive for mixed sero-groups. Six samples were positive for L. australis and $L$. canicola and two samples were positive for $L$. australis and L. Djsimea. L. canicola was the most predominant single strain observed in the study population. Amongst the mixed infections L. australis and $L$. canicola were predominantly observed. Among these strains, L. Interrogans serovar L. canicola is the most important one due to its high infection rate in dogs.

Table 1: Categorization of dogs according to type of vaccination and their habitats

\begin{tabular}{llll}
\hline Type of vaccination & Indoor & Out door & Total \\
\hline Primary vaccination & $16(76.2 \%)$ & $05(23.8 \%)$ & 21 \\
Regularly vaccinated & $20(51.28 \%)$ & $19(48.71 \%)$ & 39 \\
Randomly vaccinated & $08(38 \%)$ & $13(62 \%)$ & 21 \\
Never vaccinated & $24(41.3 \%)$ & $34(58.6 \%)$ & 58 \\
Indoor/outdoor & $68(48.9 \%)$ & $71(51.1 \%)$ & 139 \\
\hline
\end{tabular}

According to the study, statistically significant number of primarily vaccinated dogs was in indoor habitats and randomly vaccinated dogs were in outdoor habitats $(p \leq 0.05)$. That trend was not observed in regularly vaccinated and unvaccinated dog population $(p \geq 0.05)$. There was no significance difference observed in unvaccinated and regularly vaccinated dogs and they were equally distributed in indoor as well as in outdoor habitats (Table 1).

\section{DISCUSSION}

High antibody titres for leptospira were observed in domestic unvaccinated dogs indicating an acute, past or convalescent stage of infection. Leptospirosis is a highly infectious zoonotic disease and unvaccinated domestic canine population is likely to get infected in their own environments and contribute to the increasing incidence of leptospirosis in man as well as in other domestic mammals. In suburban settings in Sri Lanka there may be very high probability of domestic dogs to intermingle with stray dog populations and other peridomestic animals such as mongoose, bandicoots which are commonly observed in the area. In suburban settings in Sri Lanka there is a requirement to encourage dog owners to immunize their dogs against leptiospirosis since people have very close contact with their pet animals (Thammitiyagodage, 2011). A study conducted in Michigen, USA has indicated high prevalence of leptospirosis in dogs. Out of $1241 \mathrm{dogs}$ enrolled in their study, all the dogs were positive for at least one strain of leptospira antibody revealing previous exposure to the organism (Stokes et al., 2007).

According to the current study, predominantly observed strains in vaccinated and unvaccinated dog 
population in Sri Lanka are L. canicola, L. australis, L. Icterohaemorragica and L.djsiman. A study conducted in Thamilnadu, India have shown that $57 \%$ vaccinated and unvaccinated dogs were positive for one or more serovars of leptospira and predominantly observed strains were $L$. icterohaemorrhagiae, L. canicola, L. Pomona and L. Gripotyphosa (Senthil, 2013). Another study conducted in India has shown that the most common serova identified in clinically suspected cases of leptospira was $L$. pyrogen (Patil, 2014). Though dogs in Thamilnadu are vaccinated using inactivated Leptospira interogans serova Icterohaemorrhagiae and Canicola, antibodies for different strains using MAT test were identified in vaccinated dog population. It is reported that multiple serovas being circulated among vaccinated and unvaccinated dogs worldwide (Senthil, 2013). According to a sero-survey performed in Washington, USA, 22 out of 115 healthy dogs had antibody titres $\geq 1: 100$ to any serovar suggesting that the potential for exposure in Washington state is not uncommon (Davis, 2008). These studies give some insight to the preliminary study carried out in Sri Lanka. Study conducted in New Zealand with 466 apparently healthy dogs has shown that the serovar Copenhageni was more common (O'Keefe et al., 2002). These results clearly show that there is a geographical variation in the pattern of strains available in different regions.

Significant number of primarily vaccinated dogs was living in indoor habitats which mean that dog owners protect them when they are young and significantly higher number of randomly vaccinated dogs living in outdoor habitats may be due to dog owners neglecting their dogs when they grow up. This indicates that dog owners think when dogs are regularly vaccinated they could be let free as they are adequately protected after vaccination and unvaccinated dogs needs to be kept indoors as they are not protected from the diseases.

In Sri Lanka, usually dog owners take their dogs to private veterinarians to immunize them as there is no government programme to vaccinate them regularly. Among the vaccinated population $17.64 \%$ of the regularly vaccinated dogs and $36.8 \%$ of randomly vaccinated dogs were negative for leptospirosis antibodies using MAT. This could be due to a lack of systematic vaccination programme in the study population or unable to sero convert the animals in the selected dog population. It is necessary to carry out more systematic studies among vaccinated populations to identify the causative reasons. Small mammals are considered as the most important reservoirs of infection with large herbivores as additional significant sources of infection (Haake and Levett, 2015).

\section{CONCLUSION}

Unvaccinated dogs had high antibody titres indicating that they are exposed to the disease. These dogs may excrete leptospira spirochetes in their urine and can be potential carriers of the disease. According to this preliminary study randomly vaccinated dogs had less antibody titres than regularly vaccinated dogs. As such regular vaccination is essential to have better antibody titres among the dog population and protect them from getting the infection. As there is no distinct correlation observed in vaccination pattern and dog habitats more systematic in-depth analysis of dog population with their antibody titres is required.

\section{ACKNOWLEDGEMENT}

Authors thank MRI financial grant and veterinary surgeons from See pet Animal Clinic, Pellawatta, Fauna Animal Clinic, Battaramulla, Pet Vet Clinic, Longdon Place, Colombo, Rajagiriya Animal Clinic, Animal Clinic, Koswatta for their support extended to complete the questionnaire based study and for collection of blood samples. Special thank goes to Dr Renuka Jayatissa, Consultant Medical Nutritionist from the MRI for the assistance given in statistical analysis.

\section{REFERENCES}

Adler, B. L M., Seemann T., Murray G. L. (2011). Pathogenesis of leptospirosis: The influence of genomics. Veterinary microbiology, 153: 1-2, 7381. http//doi: 10.1016/j.vetmic.2011.02.055.

Bulach, D.M., Zuerner, R.L., Wilson, P., Seemann, T., MacGrath, A., Cullen, P.A., Davis, J., Johnson, M., Kuczek, E., Alt, D. P., Peterson-Burch, B.,Coppel, R. L., Rood, J.I., Davis, J.K., Alder, B. (2006). Genome reduction in Leptospira borgpetersenii reflects limited transmission potential. Proceedings of National Academy of Science, 103 (39):14560-5. http:// doi: 10.1073/pnas.0603979103

Blood, D.C., Radostits, O.M., (1989). Veterinary Medicine, Textbook of the Diseases of Cattle, Sheep, Pigs, Goats and Horses. Oval Road, London: BailliereTindall Ltd., 758-759.

Barragan, V., Nathan, N., Paul, K., Talima, P. (2017). Meta-analysis to estimate the load of Leptospira excreted in urine: beyond rats as important sources of transmission in low-income rural communities. BMC Research Notes, 10(71): 2384-4. Http://doi:10.1186/ s13104-017-2384-4

Davis, M.A., Evermann, J.F., Petersen, C.R., Vander Schalie, J., Besser, T.E., Huckabee, J., Daniels, J.B., Hancock, D. D., Leslie, M., Baer, R. (2008). Serological survey for antibodies to Leptospira in dogs and raccoons in Washington State. Zoonoses and Public Health, 55(8-10): 436-442. http:// doi: 10.1111/j.18632378.2008.01137.x

Evangelista, K.V., Coburn, J. (2010). Leptospira as an emerging pathogen: a review of its biology, pathogenesis and host immune responses. Future Microbiology, 5 (9), 1413-1425. http://doi: 10.2217/fmb.10.102

Greenwood, D., Slack, R.C.B., Peutherer, J.F. (2007). A Guide to microbial infections: pathogenesis, immunity, laboratory diagnosis and control (17 $7^{\text {th }}$ ed.). UK: 
Churchill Livingstone, Medical Division of Longman Group.38:369-375

Haake, D.A., Levett, P.N. (2015). Leptospirosis in Humans. Current Topics in Microbiology Immunology, 387(10): 65-97. http://doi: 10.1007/978-3-662-45059-8_5

Nelson, R.W., Couto, C.G (2004). Manual of Small Animal Internal Medicine. Westline Industries Drive, St.Louis, Missouri:Elsevier Mosby.

O'Keefe, J.S., Jenner, J.A., Sandifer, N.C., Antony, A., Williamson, N.B. (2002). Aserosurvey for antibodies to Leptospira in dogs in the lower North Island of New Zealand. New Zealand Veterinary Journal, 50(1): 23-25. http://doi:10.1080/00480169. 2002.36245

Patil, D., Dahake, R.,Roy, S., Mukherjee, S., Chowdhary, A., Deshmukh, R. (2014). Prevalence of Leptospirosis among dogs and rodents and their possible role in human leptospitosis from Mumbai, India. Indian Journal of Medical Microbiology, 32(1), 64-67. http:// doi: 10.4103/0255-0857.124319

Radostits, O. M., Blood, D.C., Gay, C. C. (1994).Veterinary Medicine: A Textbook of the Diseases of Cattle, Sheep, Pigs, Goats and Horses $\left(8^{\text {th }}\right.$ ed.). Oval Road, London: Bailliere Tindall Ltd (pp. 884-885)

Sykes, J .E., Hartmann.K., Lunn, K.F., Moore, GE., Stoddard, R.A., Goldstein, R.E. (2011). Small Animal Consensus Statement on Leptospirosis: Diagnosis, Epidemiology, Treatment, and Prevention. Journal of Veterinary Internal Medicine, 25(1), 1-13. http://doi: 10.1111/j.19391676.2010.0654.X

Senthil, N.R., Palanivel, K.M., Rishikesavan, R. (2013). Seroprevalence of Leptospiral Antibodies in Canine Population in and around Nammakkal. Journal of Veterinary Medicine, 2013: 1-4. http://dx,doi.org/10.1155/2013/971810
Stokes, J. E., Kaneene, J. B., Schall, W. D., Kruger, J. M., Miller, R., Kaiser, L., Bolin, C.A. (2007).Prevalence of serum antibodies against six Leptospira serovars in healthy dogs. Journal of the American Veterinary Medical Association, 230 (11): 1657-1664. http://doi:10.2460/javma.230.11.1657

Smythe, L., Dohnt, M., Norris, M., Meegan, S. (1993).Test method manual on leptospirosis (version 1).,WHO/FAO collaborative centre for reference and research on leptospirosis, Brisbane. 1-24.

Thammitiyagodage, M. (2011). Domestic Canine Vaccination pattern and methods of raring them in suburban setting in Colombo Sri Lanka. RIACON 2011. Proceedings of $3^{\text {rd }}$ International Conference of Rabies in Asia (RIA) Foundation, Colombo, Sri Lanka, 191-193

Victoriano, A. F. B., Lee, D., Nina, GB., Lolita, L., Takeshi, K., Khanchit, L., Bee, L. O., Gyanendra, G., Hall, J., Coulombe, C. A., Yanagihara, Y., Yoshida, S. I., Adler, B. (2009). Leptospirosis in the Asia and Pacific region. BMC Infectious Diseases, 9: 147. http://DOI: 10.1186/1471-2334-9-147

Vijayachari, P., Sugunana, A.P.,Shriram,A. (2008). Leptospirosis: an emerging global public health problem. Journal of Bio Science, 33(4):557-69.

World Health Organization (2003). Human leptospirosis: guidance for diagnosis, surveillance a n d control. Retrieved from http://whqlibdoc.who.int/hq/2003/WHO_CDS_CSR EPH_2002.23.pdf. 